\title{
Hip instability: a review of hip dysplasia and other contributing factors
}

\author{
Matthew J. Kraeutler ${ }^{1}$ \\ Tigran Garabekyan² \\ Cecilia Pascual-Garrido1 \\ Omer Mei-Dan'1 \\ 1 University of Colorado School of Medicine \\ 2 Southern California Hip Institute \\ Corresponding author: \\ Omer Mei-Dan \\ Hip Preservation/Sports Medicine/Orthopedics \\ University of Colorado \\ 12631 East 17th Avenue \\ Mail Stop B202, Room L15-4505 \\ Aurora, Colorado 80045 (USA) \\ E-mail: omer.meidan@ucdenver.edu
}

\section{Summary}

Background: Hip instability has classically been associated with developmental dysplasia of the hip (DDH) in newborns and children. However, numerous factors may contribute to hip instability in children, adolescents, and adults.

Purpose: This review aims to concisely present the literature on hip instability in patients of all ages in order to guide health care professionals in the appropriate diagnosis and treatment of the various disorders which may contribute to an unstable hip.

Methods: We reviewed the literature on the diagnosis and surgical management of hip dysplasia and other causes of hip instability.

Conclusions: Multiple intra- and extra-articular variables may contribute to hip instability, including acetabular bony coverage, femoral torsion, femoroacetabular impingement, and soft tissue laxity. Physical examination and advanced imaging studies are essential to accurately diagnose the pathology contributing to a patient's unstable hip. Conservative management, including activity modification and physical therapy, may be used as a first-line treatment in patients with intra-articular hip pathology. Patients who continue to experience symptoms of pain or instability should proceed with arthroscopic or open surgical treatment aimed at correcting the underlying pathology.

Level of evidence: V.

KEY WORDS: developmental dysplasia of the hip, femoral anteversion, femoroacetabular impingement, hip instability, periacetabular osteotomy.

\section{Introduction}

Hip instability is a diagnosis with numerous etiologic factors including, most notably, developmental dysplasia of the hip (DDH). Based on universal screening, the incidence of DDH is very low, with approximately $0.24 \%$ of newborns demonstrating ultrasonographic findings of DDH within the first two postnatal weeks ${ }^{1}$. When hip instability or subluxation persists in newborns beyond three weeks of life, conservative treatment with use of a Pavlik harness is indicated ${ }^{2}$. If the hip remains unstable following six weeks of treatment with a Pavlik harness, closed reduction and casting should be attempted. If concentric reduction cannot be obtained using these methods, the patient should undergo open reduction of the hip in conjunction with a varus derotational femoral osteotomy. Beyond the age of three years, the remodeling capacity of the shallow acetabulum is insufficient and an acetabular realignment osteotomy is typically indicated to restore stability.

Recent advances in the pathomechanics of hip instability have implicated several factors, in addition to $\mathrm{DDH}$, as contributing to hip instability in the adolescent and young adult population. As such, the definition of hip instability must be broadened to encompass other bony and soft tissue contributors. The purpose of this review is to detail the various features of hip instability, to describe the appropriate clinical and radiographic evaluation of patients with hip instability, and to present recommendations for conservative or surgical management of symptomatic hip instability based on the current literature and our own opinions based on experience.

This article submits to the ethical standards of the journal ${ }^{3}$.

\section{Features of hip instability}

Various factors may underlie the clinical diagnosis of hip instability, including acetabular bony coverage, femoral torsion ${ }^{4}$, femoroacetabular impingement (FAI)-induced instability ${ }^{5}$, as well as soft tissue laxity ${ }^{6}$. In DDH, acetabular coverage is typically deficient anteriorly and superolaterally giving rise to a decrease in the lateral center edge angle (LCEA) and an increase in the acetabular anteversion. An LCEA between 20 and $25^{\circ}$ indicates borderline dysplasia whereas an LCEA $<20^{\circ}$ represents frank dysplasia. Similarly, the normal acetabular version of $15-20^{\circ}$ is 
increased in the setting of dysplasia to values greater than $20^{\circ}$. Corrective osteotomy is typically indicated when the LCEA is less than $20^{\circ}$ and/or the acetabular version is greater than $30^{\circ}$. There are, however, certain variants of DDH characterized by either global acetabular deficiency or posterolateral deficiency for which the acetabulum may have normal version or retroversion (Figure 1). Previous studies have reported retroversion in up to $18 \%$ of dysplastic hips, with a corresponding radiographic crossover sign due to posterior deficiency rather than anterior overcover$\operatorname{age}^{7,8}$. Given that acetabular version is a measurement of anterior coverage relative to posterior coverage, globally reduced coverage may leave version unchanged. A more direct method of assessing anterior coverage is by evaluating the degree of overlap between the anterior wall and the femoral head (Figure 1b).

The acetabular sourcil morphology may also provide clues to underlying instability mechanics in a hip with otherwise borderline or normal LCEA and acetabular version. A flattened sourcil or one that slopes up laterally may indicate a mildly incongruous femoroacetabular joint that can contribute to instability ${ }^{9}$. Measurement of lateral coverage in these cases should be made to the base of the upsloping portion of the sourcil as the bone lateral to this location does not contribute to weight-bearing or stability. Additionally, normal lateral coverage indices should be interpreted with caution in the setting of a flattened sourcil that lacks the normal concavity important for a stable femoroacetabular articulation.

Femoral torsion is an important factor when evaluating hip stability. Excessive femoral antetorsion creates functional undercoverage of the femoroacetabular joint anteriorly and may precipitate instability symptoms even in the setting of normal or borderline acetabular coverage (Figure 2). Femoral torsion should be evaluated independently and the combined index of femoral torsion + acetabular version (COTAV index) utilized in decision-making regarding hip stability and the need for corrective realignment procedures ${ }^{10}$.

The radiographic assessment of acetabular coverage, acetabular version, and femoral torsion is described in greater detail under the heading "Radiographic characterization".

In addition to the above-mentioned causative factors, hip instability is associated with several characteristic intra- and extra-articular abnormalities typically due to chronic overload of secondary soft tissue stabilizers (Table I). With regard to intra-articular abnormalities, both labral length (measured with magnetic resonance imaging) ${ }^{11}$ as well as labral width (measured during arthroscopy) $)^{12}$ are larger in patients with reduced lateral acetabular coverage. Labral enlargement is thought to represent adaptive hypertrophy in the setting of increased shear forces within the unstable hip joint, though it may also be due to incomplete ossification of the cartilaginous acetabulum during hip development.

Articular cartilage thickness has also been shown to exhibit hypertrophy, particularly in the acetabular roof, in newborns with $\mathrm{DDH}^{13,14}$. Adults with borderline or frank dysplasia show a similar trend of femoroacetabular cartilage hypertrophy ${ }^{15}$, which is thought to arise as a compensatory mechanism to weight-bearing and sheer stress. The same may be seen in pediatric patients with DDH after hip reduction by decreasing the medially directed force of the femoral head into the acetabulum ${ }^{13}$. The lack of bony coverage places the enlarged labrum in a weightbearing position rendering it susceptible to tears. Similarly, the thickened articular cartilage is exposed to increased shear forces resulting in a characteristic "inside-out" type articular cartilage flap tear, reported in $50 \%$ of dysplastic patients by one group ${ }^{16}$

The ligamentum teres (LT) is an important stabilizer of the hip joint, particularly in patients with osseous instability, such as inferior acetabular insufficiency, borderline or frank hip dysplasia, or $\mathrm{FAI}^{17}$. Tears of the LT may result from chronic overload in the setting of hyperlaxity, instability, or chronic irritation such as that which occurs in patients with FAI.

Hip instability is also associated with extra-articular pathologies, such as iliopsoas tendinitis due to compensatory loading ${ }^{18,19}$. In dysplasia, the iliopsoas rests directly against the anterior femoral head and acts as secondary dynamic stabilizer leading to overload, inflammation, and pain. Patients with increased femoral anteversion may also have an increased risk of lateral patellofemoral dislocation 20 . From our experience, we have also noticed an association between hip instability symptoms and tendinitis of the hamstrings, which we believe occurs to maintain sagittal pelvic load balance.

Table I. Intra- and extra-articular abnormalities associated with hip instability.

\begin{tabular}{ll}
\hline Intra-articular & Extra-articular \\
\hline Reduced acetabular coverage & Iliopsoas tendinitis \\
Femoroacetabular impingement & Sacroilitis \\
Ligamentum teres tears & Osteitis pubis \\
Increased labral length and width & Patellofemoral dislocation \\
Acetabular cartilage hypertrophy & Hamstring tendinitis \\
\hline
\end{tabular}




\section{Clinical evaluation}

On physical examination, patients with hip instability typically present with an apprehensive gait due to avoidance of terminal extension. Passive hip rotational range of motion (ROM), both at neutral and $90^{\circ}$ of hip flexion, is strongly correlated with femoral and acetabular version and should be taken into consideration when formulating a treatment algorithm ${ }^{10}$. Patients with FAI will typically have markedly decreased internal rotation $\left(0-15^{\circ}\right)$ and flexion $\left(<110^{\circ}\right)$ due to bony impingement. However, patients with a cam lesion in the presence of hip dysplasia or other forms of instability will have greater internal rotation than would be expected given the cam lesion alone.

The log roll test is frequently used during the hip examination as pain during this test may indicate inflammation or capsulitis within the hip joint, confirming intra-articular pathology as the source of the patient's complaints. In patients with instability, the log roll test may indicate capsular laxity if excessive passive external rotation is noted by the examiner or if there is loss of normal log roll recoil of the hip ${ }^{21}$. The anterior apprehension test may also be used to test for hip dysplasia. The patient is positioned supine with the unaffected hip flexed and affected hip extended, and the examiner abducts and external rotates the affected hip. A positive test is indicated by the presence of apprehension or subluxation during this maneuver. This test may alternatively be performed in the lateral position by abducting, extending, and externally rotating the affected hip while applying an anteriorly directed force on the proximal thigh. Finally, the "LT test" may be used to determine if there exists a partial rupture of the ligamentum teres ${ }^{22}$. This test is performed with the patient in $70^{\circ}$ of hip flexion and $30^{\circ}$ short of full hip abduction. The examiner internally and externally rotates the hip to its limits, with pain during internal or external rotation indicating a positive test. The sensitivity and specificity of this test for predicting an LT tear is $90 \%$ and $85 \%$, respectively, based on arthroscopic findings 22 .

Joint hypermobility due to soft tissue laxity, either idiopathic or due to Ehlers-Danlos syndrome ${ }^{6}$ or Marfan syndrome 23 , may be assessed with the Beighton Hypermobility Score 24,25 . This score is based on a scale from 0 to 9 , with each of the following counting as 1 point: hyperextension of the right/left elbow, hyperextension of the right/left knee, excessive passive dorsiflexion $\left(>90^{\circ}\right)$ of the right/left fifth metacarpophalangeal joints, passive dorsiflexion of the right/left thumb to the forearm, and ability to rest the palms and hands flat on the floor during trunk forward flexion with the knees fully extended. Often, scores $\geq 4$ represent joint hypermobility ${ }^{26}$ and we interpret scores $>6$ to indicate pathologic laxity.

\section{Radiographic characterization}

Certain radiographic parameters have been developed to diagnose hip dysplasia and to assist with treatment strategies. Lateral acetabular coverage is typically assessed via the lateral center edge angle (LCEA) of Wiberg ${ }^{27}$ or using the Ogata modification ${ }^{28}$ and is measured on an anteroposterior (AP) radiograph, though it may also be measured on a computed tomography (CT) scan ${ }^{29}$. The LCEA represents the angle formed between a vertical line through the center of the femoral head (orthogonal to the horizontal pelvic axis) and a line connecting the center of the femoral head and the lateral edge of the acetabular roof or sourcil 28,30 . Patients are diagnosed with borderline hip dysplasia when the LCEA is $20-25^{\circ}$ and frank dysplasia when the LCEA $<20^{\circ}$. In a normal, asymptomatic population, a mean LCEA of $31^{\circ}$ has been measured ${ }^{31,32}$. It is important to keep in mind that an LCEA $>25^{\circ}$ does not rule out acetabular dysplasia altogether, as some patients have isolated anterior and/or posterior dysplasia resulting in symptomatic instability (Figure 1) which can be exacerbated by laxity or excessive femoral torsion. Additionally, the LCEA should be interpreted in the context of sourcil morphology, as a flattened or upsloping sourcil may be an occult sign of an incongruous and potentially unstable femoroacetabular articulation.

Anterior acetabular coverage may be measured by the anterior center edge (ACE) angle, also known as the angle of Lequesne ${ }^{33}$, which is measured on the false profile view. The ACE angle is formed between a vertical line through the center of the femoral head and a line connecting the center of the femoral head and the most anterior portion of the acetabular sour$\mathrm{cil}^{30}$. ACE angles $<20^{\circ}$ suggest bony deficiency and may lead to instability. In contrast, Frank et al. ${ }^{31}$ measured the mean ACE angle in asymptomatic patients to be $30^{\circ}$. Additionally, anterior acetabular coverage may be assessed by the degree of overlap between the anterior wall and femoral head. Hips in which the anterior wall/femoral head overlap is $<20 \%$ of the femoral head diameter are concerning for anterior instability. A more reproducible method of assessing anterior acetabular coverage is with a CT false-profile view $^{34}$. This method produces a cross-sectional image in the same obliquity as false-profile radiographs and eliminates projectional errors.

The Tönnis (or sourcil) angle is measured on AP radiographs and provides information on a patient's acetabular inclination. The angle is measured by drawing three lines: 1) a horizontal line connecting the base of the acetabular teardrops, 2) a horizontal line running parallel to line 1 , through the inferior point of the acetabular sourcil (point I), and 3) a line running from point I to the lateral edge of the acetabular sourcil. The Tönnis angle is the angle formed by the intersection of lines 2 and 3 and is considered normal at $0-10^{\circ} 30$. Tönnis angles $>10^{\circ}$ and $<0^{\circ}$ represent increased and decreased inclination, respectively, with increased acetabular inclination associated with hip instability.

In addition to acetabular inclination, acetabular version may also be assessed based on the crossover or figure-of-eight sign ${ }^{30}$. If a crossover sign is appreciated (i.e. if the anterior rim of the acetabulum crosses 


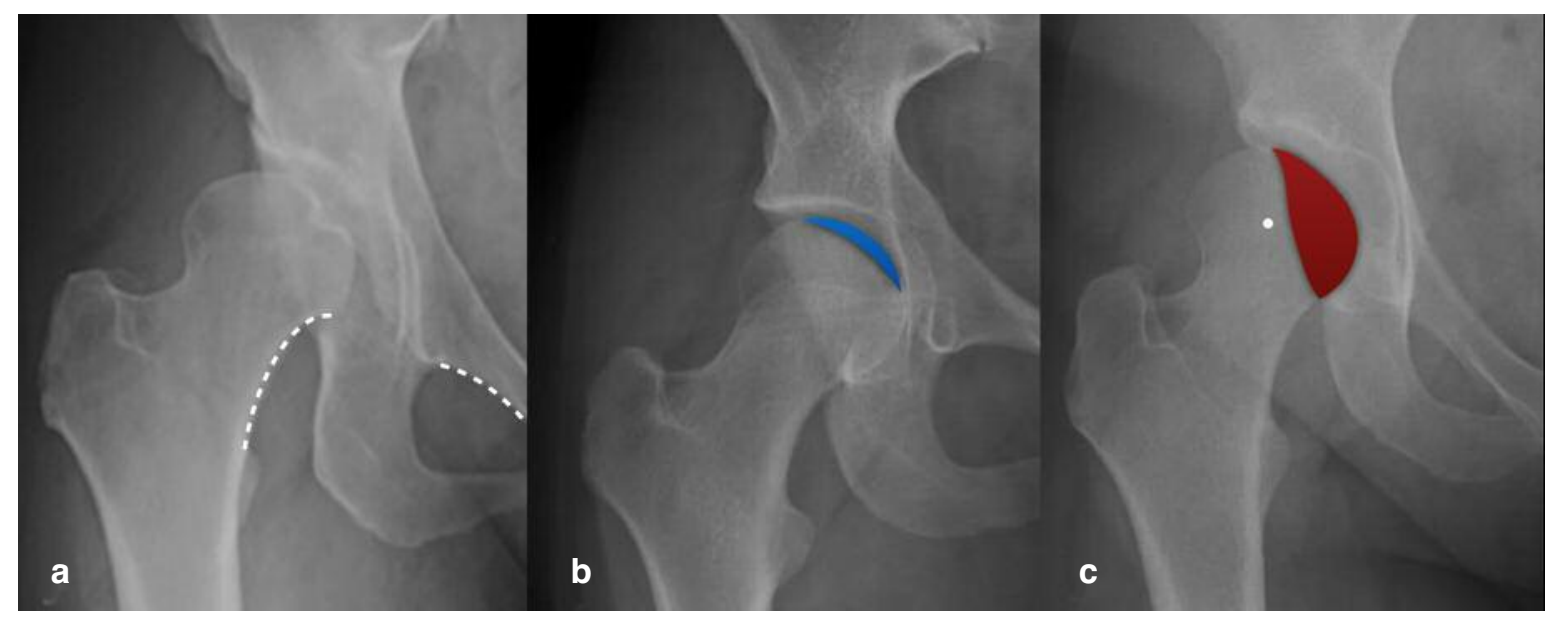

Figure 1a-c. a) Anteroposterior (AP) radiograph of a right hip with severe lateral acetabular dysplasia, superolateral migration of the femoral head, and a break in Shenton's line (dashed white line). b) AP radiograph of a right hip with borderline acetabular dysplasia, deficient anterior acetabular coverage, and preserved lateral center edge angle. The blue shaded area indicates the small degree of overlap between the femoral head and the anterior acetabular wall. Note the flattened sourcil or acetabular roof. c) AP radiograph of a right hip with severe lateral and posterior acetabular dysplasia. The red shaded area indicates the overlap between the femoral head and the posterior acetabular wall. Note that the center of the femoral head (white dot) lies lateral to the posterior wall, indicating a positive posterior wall sign with deficient posterior coverage.

the line of the posterior rim before reaching the lateral aspect of the sourcil), the acetabulum is locally or globally retroverted. Global acetabular retroversion can be associated with a posterior wall deficiency, a crossover sign, and a prominent ischial spine. It is important to note that approximately $18 \%$ of dysplastic hips have retroversion due to posterior deficiency and not due to anterior overcoverage ${ }^{8}$. Increased acetabular anteversion is a common finding among infants ${ }^{35}$ and adults $^{36}$ with DDH. Interestingly, acetabular anteversion has been associated with internal rotation of the innominate bone, which may indicate that structural abnormalities exist throughout the pelvis in patients with $\mathrm{DDH}$, rather than localized dysplasia around the hip ${ }^{36}$. For acetabular version to be correctly interpreted, there must not be excessive pelvic tilt or rotation during imaging ${ }^{37}$.

The femoral neck torsion angle (FNTA) represents the angle between the axis of the femoral neck and the coronal plane of the femoral condyles (condylar plane $)^{38}$. The term femoral anteversion refers to a femoral neck that inclines anterior to the condylar plane, while femoral retroversion indicates inclination posterior to the condylar plane. Because multiple planes must be viewed to measure the FNTA, CT reconstruction is often used to measure this parameter, though magnetic resonance imaging (MRI) ${ }^{39}$ and plain radiography with the Dunn view ${ }^{40}$ have also been used. In patients with unilateral Tönnis type IV $\mathrm{DDH}$, the dislocated hip has been shown to exhibit significant femoral anteversion compared with the unaffected hip ${ }^{35}$. In addition, femoral anteversion has been shown to associate with passive internal rotation of the hip ${ }^{10}$. Excessive femoral antetorsion creates functional anterior femoroacetabular undercoverage, and may give rise to anterior instability and il- iopsoas symptomatology, even in the setting of normal acetabular coverage. Hip instability that is driven predominantly by excessive femoral antetorsion should be addressed via derotational femoral osteotomy (Figure 2).

Imaging may also be used to detect intra-articular abnormalities that may indicate hip instability, even if the above parameters are normal or borderline normal. MRI may be used to measure labral length in the lateral, anterior, and anteroinferior portion of the acetabulum, with increased lengths in all directions being associated with deficient lateral acetabular coverage $^{11}$. MRI may also demonstrate hypertrophic acetabular cartilage and/or intra-articular fibrous tissue in patients with $\mathrm{DDH}^{13}$. MRI or magnetic resonance arthrography (MRA) may be used to assess for tears of the ligamentum teres, though MRI has been shown to have lower sensitivity and specificity in detecting partial LT tears ${ }^{41}$. These intra-articular abnormalities are useful when evaluating a patient with borderline acetabular dysplasia and deciding between minimally invasive arthroscopic treatment versus bony realignment procedures to address the instability. In these cases, every additional piece of information that may suggest occult instability can help the clinician in arriving at the correct diagnosis and treatment plan.

\section{Treatment}

\section{Conservative management}

As mentioned above, newborns diagnosed with $\mathrm{DDH}$ by ultrasound findings should begin conservative treatment with a Pavlik harness for a minimum of two weeks ${ }^{2}$. For adolescents and adults with hip dyspla- 


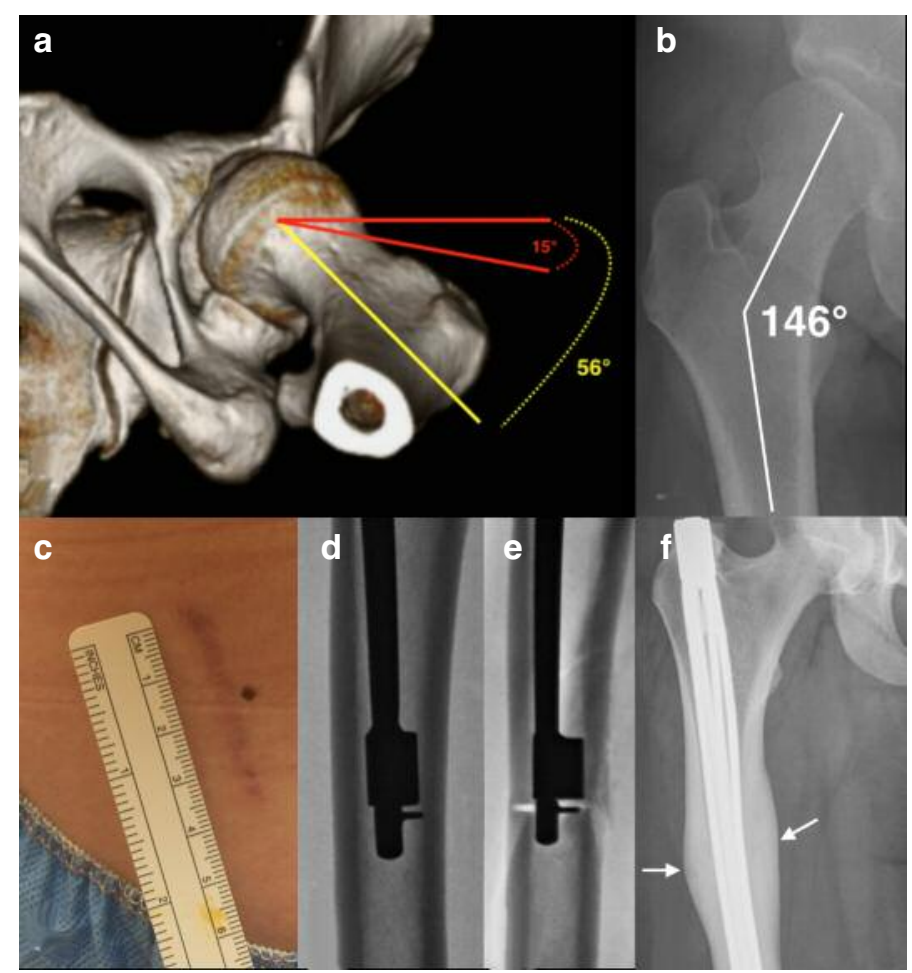

Figure 2 a-f. a) Three-dimensional reconstruction of the left hip from computed tomography (CT) images indicating excessive femoral antetorsion of 56 degrees and resultant functional undercoverage of the anterior femoral head. b) AP radiograph of the right hip in a patient with frank acetabular dysplasia and associated excessive femoral neck shaft angle of 146 degrees corresponding with coxa valga. c) Minimally invasive surgical site used to perform a derotational femoral osteotomy (DFO) measuring $4 \mathrm{~cm}$ in length. d) Intraoperative fluoroscopic radiograph of the right femur with intramedullary saw in place prior to performing femoral osteotomy. e) Intraoperative fluoroscopic radiograph of the right femur with intramedullary saw in place following completion of femoral osteotomy. f) Postoperative AP radiograph of the right femur with expandable intramedullary rod in place following DFO to correct for antetorsion and coxa valga deformities. Note the well-healed femoral osteotomy site with robust callus (white arrows) and slight varus angle across osteotomy to correct for coxa valga.

sia, a trial of conservative treatment may be indicated. This may include patient education, activity modification, physical therapy, and anti-inflammatory medications. Hunt et al. ${ }^{42}$ demonstrated that patients (mean age 35 years) with pre-arthritic, intra-articular hip pathologies, including hip dysplasia and FAI, improved following one year of conservative $(n=23)$ or operative $(n=29)$ treatment. No difference in improvement was demonstrated between the surgical and conservative groups, although patients in both groups significantly reduced their activity level during the year following treatment initiation. Patients in this study with more active lifestyles were more likely to choose surgical intervention.

In patients with $\mathrm{FAI}$, initial nonoperative treatment may be indicated, with physical therapy and activity modification conferring some benefit ${ }^{43,44}$. However, conservative treatment may not improve hip range of motion ${ }^{43}$. Intra-articular injections may also serve as an efficacious form of conservative treatment, with injections of hyaluronic acid providing safe and effective pain relief ${ }^{45,46}$.

\section{Surgical management}

Surgical treatment of hip instability may be divided into two general categories: soft-tissue procedures and bony realignment. Surgical treatment using a soft-tissue approach may include capsular plication or capsulorrhaphy in patients with capsular laxity or iatrogenic hip instability 47,48 . The iliofemoral ligament (IFL) is recognized as the dominant stabilizer of the anterior hip. Our group recently described IFL reconstruction with an Achilles tendon allograft for patients with recurrent hip instability due to anterior capsular deficiency ${ }^{49}$.

The iliopsoas tendon is also recognized as an important dynamic hip stabilizer, and iliopsoas tenotomy or arthroscopic psoas lengthening may contribute to iatrogenic hip instability and inferior clinical outcomes $^{50,51}$. Sansone et al. ${ }^{52}$ published a case report of two patients who suffered non-traumatic hip dislocations following arthroscopic capsulotomy and iliopsoas tenotomy. Therefore, iliopsoas tenotomy or lengthening procedures should be avoided in patients with hip instability and concomitant iliopsoas tendinitis or psoas-associated snapping hip syndrome.

Labral tears may also contribute to hip instability. Nepple et al. ${ }^{53}$ have shown that the labrum represents the primary hip stabilizer to distraction forces at small displacements (1-2 mm). In patients with borderline hip dysplasia, arthroscopic capsular plication and labral repair has resulted in favorable outcomes $^{54}$. In patients with soft tissue laxity due to Ehlers-Danlos syndrome, capsular plication and labral repair may lead to significant improvement in subjective hip stability ${ }^{6}$. Uchida et al. ${ }^{55}$ analyzed predictors of worsened outcomes in patients with $\mathrm{DDH}$ undergoing arthroscopic management with labral repair and capsular closure. These predictors included a femoral neck-shaft angle $>140^{\circ}$, a LCEA $<19^{\circ}$, and a body mass index (BMI) $>23 \mathrm{~kg} / \mathrm{m}^{2}$. In patients presenting with these predictors, an osteotomy procedure may result in better outcomes.

Bony realignment is a more invasive approach and includes periacetabular osteotomy (PAO) and/or derotational femoral osteotomy (DFO). Hip dysplasia 
frequently results in secondary hip osteoarthritis $(\mathrm{OA})^{56}$. However, young patients are not ideal candidates for total hip arthroplasty (THA). A PAO allows for mechanical correction of hip dysplasia by altering the patient's pelvic and hip joint anatomy without replacing the hip. A PAO is performed by creating three periacetabular osteotomies and a controlled fracture in order to completely mobilize the acetabulum from the remaining pelvis ${ }^{57}$. This allows for acetabular reorientation to provide greater coverage of the femoral head. Our preference is to use the Birmingham Interlocking Pelvic Osteotomy (BIPO) which creates an inherently more stable construct and allows for immediate full weight-bearing postoperatively (Figure 3$)^{58}$. Meanwhile, the posterior column of the true pelvis remains intact, which maintains stability through an intact pelvic ring. Based on a systematic review, Lodhia et al. ${ }^{59}$ found that arthroscopic treatment has resulted in improved outcomes in patients with borderline dysplasia (LCEA between $18-25^{\circ}$ ), while PAO has mostly been used successfully in patients with frank hip dysplasia. Despite these generalities, borderline dysplasia must be approached with caution as many patients are excellent candidates for PAO or DFO given enough markers of instability. Borderline dysplastic patients are treated on a case-by-case basis and the integrity of the articular cartilage is an important factor in the treatment algorithm.
The hip survival rate following a periacetabular osteotomy has been measured at $81.6 \%$ at a mean 9.2 years follow-up 60 and $60 \%$ at a mean 20.4 years follow-up ${ }^{57}$. Severe dysplasia, increased age at surgery, and increased OA score (Tönnis grade) prior to surgery are some of the factors that have been associated with an increased risk of conversion to total hip replacement 57,60 . After matching for age and gender, a recent study ${ }^{61}$ showed that patients (average age 56.5 years) who undergo THA after a failed PAO have worse outcomes (Harris Hip Score, ROM) compared to patients who undergo primary THA for hip dysplasia. Kalore et al. ${ }^{62}$ recently showed that, in middle-aged patients (30-50 years), PAO is associated with longer durations of pain and gait aids compared with THA, though either of these procedures can be expected to improve sports participation and function to comparable levels. However, the minimum follow-up was only four years in this study, and younger patients undergoing primary THA would likely need at least one revision in their lifetime. Furthermore, it is important to understand the differences in recommended athletic pursuits after each surgical option. Arthroplasty will require the abandonment of all impact-loading activities (running, contact sports, tennis, skiing) while PAO, once healed, has no associated restriction on activity level.

Derotational femoral osteotomy is indicated for pa-

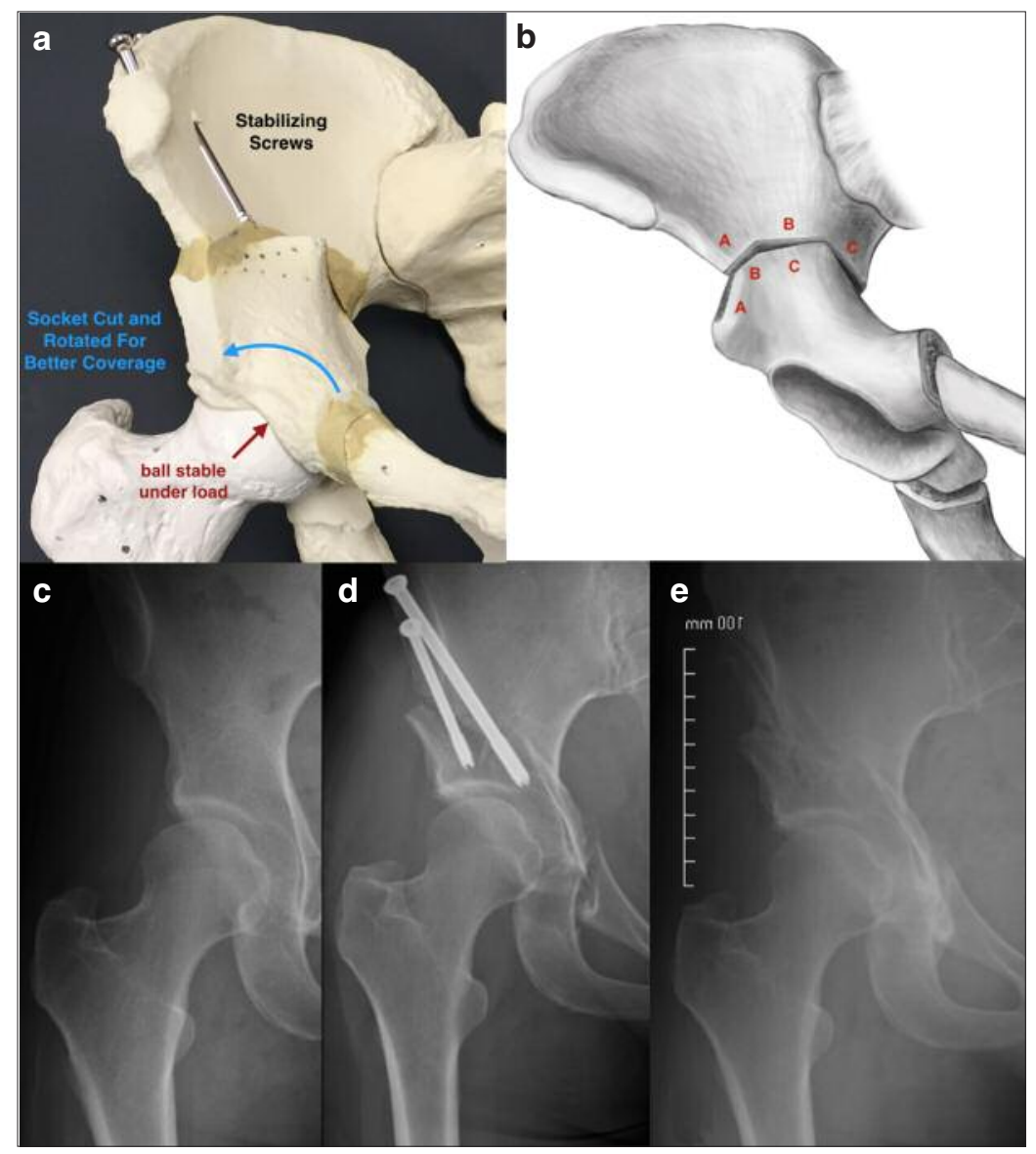

Figure 3 a-e. a) Model representation of a periacetabular osteotomy (PAO) indicating the rotational correction of the acetabular fragment (blue arrow) which creates normalized femoroacetabular joint contact forces (red arrow) and reduces superolaterally-directed shear forces creating symptomatic instability. b) Schematic diagram of the Birmingham Interlocking Pelvic Osteotomy (BIPO) indicating location of ilium cuts ( $a, b$, and $c)$ and interlocking construct following rotation of central acetabular fragment. This osteotomy is stable enough for immediate full weight-bearing and, as a result, results in less muscle atrophy and deconditioning. c) AP radiograph of a right hip with borderline acetabular dysplasia predominantly due to lateral and posterior deficiencies in coverage. Note the short and inclined acetabular roof or sourcil. d) AP radiograph of a right hip following PAO with cannulated screw fixation. Note the degree of posterolateral coverage gained by the procedure with normalization of the relationship between the anterior and posterior walls. e) AP radiograph of a right hip following screw removal from healed PAO. 
tients with significant symptomatic femoral anteversion $\left(>40^{\circ}\right)$ or retroversion $\left(<-10^{\circ}\right)$ (Figure 2). Patients with femoral anteversion (FA) often walk with an internal foot progression angle and exhibit symptoms of hip instability due to functional anterior undercoverage of the femoral head, while those with significant femoral retroversion often walk with an external foot progression angle and exhibit symptoms of concomitant FAI. The majority of cases of FA spontaneously correct by age $8^{63}$. Therefore, DFO is indicated in patients over the age of 8 with symptoms resulting from excessive FA. DFO may be performed using an intertrochanteric or supracondylar osteotomy. The intertrochanteric method has been shown to result in a lower complication rate, more accurate correction of the intoeing deformity, and a reduced need for postoperative immobilization 64 . Despite altering the rotational alignment of the femur, the DFO procedure does not significantly alter the lengths of the hamstrings or adductors ${ }^{65}$ but does result in extensive rehabilitation due to change in muscle direction and function.

\section{Arthroscopic evaluation}

During hip arthroscopy, it is important to evaluate for any articular cartilage or labral injuries resulting either from a primary injury or as a secondary insult due to underlying pathology. The patterns of articular cartilage and labral injuries differ between patients with FAI and hip dysplasia, and therefore the intra-articular injuries may aid in the determination of the nature of hip pathology. Kaya et al. ${ }^{66}$ performed a retrospective review of 100 consecutive hip arthroscopies and noted a high incidence of full-thickness cartilage defects in the anterior superior and middle superior zones of the acetabulum in patients with FAl and borderline dysplasia. In patients with joint laxity, partialthickness defects (grade 1 and 2) were dominant. Finally, in patients with acetabular dysplasia, full-thickness defects extended to the posterior superior zone. The incidence of full-thickness defects of the femoral head was higher in patients with FAI and borderline dysplasia compared to those with joint laxity and acetabular dysplasia.

In another retrospective review, Tamura et al. ${ }^{67} \mathrm{com}-$ pared differences in labral tear morphology between patients with FAI, mild dysplasia (LCEA between 0$\left.20^{\circ}\right)$, and severe dysplasia ( $\left.\operatorname{LCEA}<0^{\circ}\right)$. Patients with $\mathrm{FAl}$ and mild dysplasia were more likely to have labral tears in the anterior and anterosuperior zones, whereas those with severe dysplasia were more likely to have tears in the anterosuperior and superior zones. Tears associated with the base of the labrum were more common in patients with FAI, whereas tears associated with the body of the labrum were more common in those with severe dysplasia. Furthermore, the frequency of articular cartilage damage adjacent to labral base tears was significantly higher than cartilage damage adjacent to labral body tears. The pattern of acetabular articular cartilage flap tears has also been investigated for instability and FAI, with characteristic differences in tear morphology. Whereas cam FAl typically results in an "outside-in" pattern of articular cartilage damage, with a break in the chondrolabral junction and a centrally based flap ${ }^{68}$, hip instability has been correlated with an "inside-out" pattern of cartilage damage, with preservation of the chondrolabral junction ${ }^{16}$ (Figures 4, 5). This difference in articular cartilage injury is thought to stem from the inside-out shearing mechanism of injury in unstable hips as compared with the outsidein impingement mechanism of FAI. We are currently investigating whether the pattern of articular cartilage injury is predictive of failure following isolated soft-tissue surgery in the setting of borderline dysplasia. Several Authors have advocated for arthroscopic treatment of borderline dysplasia noting good to excellent short-term outcomes 54,69 . However, in our experience, this soft tissue-only approach yields deteriorating results after 6-12 months in patients who have a predominantly "inside-out" pattern of articular cartilage injury. We speculate that the good to excellent outcomes in the aforementioned studies are likely due to an "outside-in" pattern of injury in their cohorts of FAI patients who have borderline dysplasia. Additionally, the improvements seen in patient-based outcome measures are lower in these cohorts with borderline dysplasia when compared to arthroscopic treatment of FAl without dysplasia.

Tears of the ligamentum teres may also contribute to hip instability, particularly in patients with osseous instability ${ }^{17}$. LT tears may not always be appreciated on preoperative imaging, so it is important to assess for these injuries during arthroscopy. Botser et al. ${ }^{70}$ found LT tears in 284 of 558 patients (51\%) undergoing hip arthroscopy for various pathologies. Overall, $3.7 \%$ of patients had a complete rupture, $43 \%$ had a partial tear, and $4.5 \%$ had a degenerative tear. In another study ${ }^{71}$, the ligamentum teres was found to be normal in 152 of 184 (83\%) patients undergoing hip arthroscopy for various hip pathologies. However, complete ruptures were found to be present in $7 \mathrm{pa}$ tients $(3.8 \%)$, a prevalence nearly identical to that cited by Botser et al. ${ }^{70}$. Patients with partial/degenerative tears or complete rupture of the LT may undergo LT debridement or reconstruction, respectively. LT reconstruction is typically indicated only for complete ligament tears. The majority of techniques for LT reconstruction employ femoral tunnel drilling through the peritrochanteric region and differ mainly with regard to graft type or acetabular fixation. In contrast, our group recently described an arthroscopic technique for reconstruction of the ligamentum teres with graft fixation at the femoral head-neck junction ${ }^{72}$.

\section{Conclusions}

Although hip instability is classically been associated with developmental dysplasia of the hip, multiple other factors may contribute to an unstable hip. In addition to acetabular bony coverage, these factors in- 


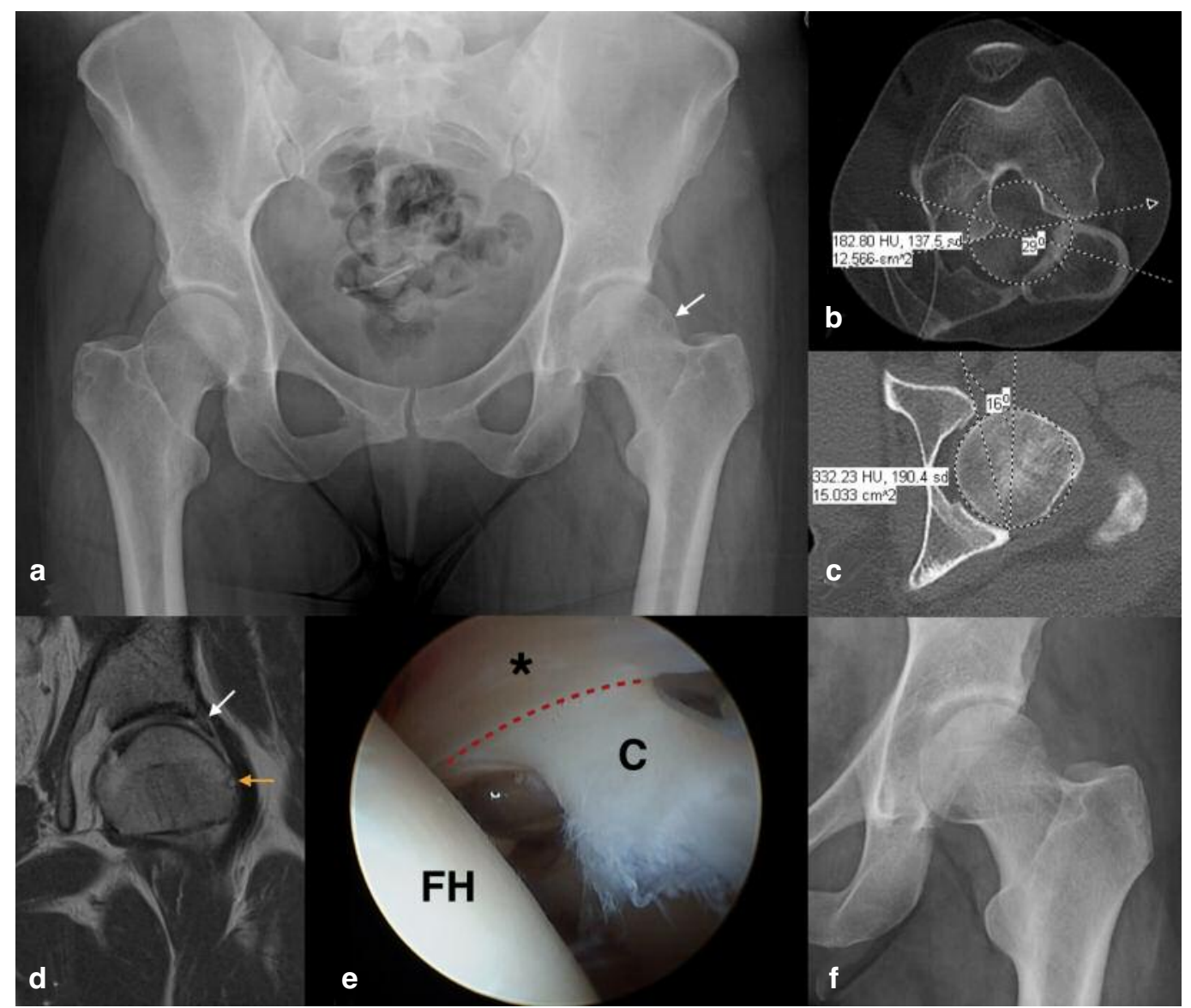

Figure 4 a-f. a) AP radiograph of a pelvis in a 36-year-old female patient presenting with 6 month history of left groin pain and mechanical symptoms, having failed conservative management. She has a history of childhood developmental dysplasia of the hip $(\mathrm{DDH})$ and her clinical examination is concerning for ligamentous laxity with a Beighton score of 7 as well as left hip internal rotation of $30^{\circ}$ with the hip at $90^{\circ}$ of flexion, despite having a large cam deformity (white arrow). Note the positive posterior wall sign with slight posterior acetabular deficiency. b) Axial CT scans of the left hip with overlapping images through the center of the femoral head, the lesser trochanter, and the distal femur. Femoral torsion measures $29^{\circ}$ antetorsion (normal $10-20^{\circ}$ antetorsion). c) Axial CT scan of the left hip through the center of the femoral head indicating equatorial acetabular version to be $16^{\circ}$ anteversion (normal $15-20^{\circ}$ anteversion). Given the posterior wall deficiency, this value underrepresents the true extent of anterior acetabular deficiency. d) Coronal proton density (PD) fat saturated (FT) image of the left hip indicating enlarged labrum (white arrow), which serves as a marker of instability. Note the large lateral cam lesion with impingement cyst in the upper femur (orange arrow). e) Arthroscopic image of the left hip demonstrating instability type "inside-out" articular cartilage flap tear (c) with preserved chondrolabral junction (red dashed line) and enlarged labrum (black asterisk). Note that this pattern of injury is not what would be expected from cam-type FAI which typically results in a break at the chondrolabral junction and an "outside-in" articular flap tear. FH = femoral head. f) Postoperative AP radiograph of the left hip demonstrating restoration of the normal femoral head-neck contour with improved offset.

clude femoral torsion, femoroacetabular impingement, and soft tissue laxity. A number of physical examination tests and radiological parameters may be used to correctly diagnose the pathology contributing to a patient's unstable hip. In distinguishing between borderline and frank hip dysplasia, the lateral center edge angle should be measured on plain radiography, with angles between $20-25^{\circ}$ indicating borderline dysplasia and angles $<20^{\circ}$ indicating frank dysplasia. Patients with intra-articular pathology contributing to hip instability may attempt a trial of conservative treatment, including activity modification and physical therapy. For patients whose symptoms continue or worsen, surgical management is indicated. Surgical treatment may include arthroscopic labral repair, capsular plication, iliofemoral ligament reconstruction, or osteotomy procedures such as a periacetabular osteotomy or a derotational femoral osteotomy. Patients with borderline hip dysplasia generally improve with arthroscopic treatment in the short term, however some experience recurrent symptoms upon initiating higher demand activities and are best treated with realignment osteotomy. Patients with frank dysplasia often require an osteotomy procedure and do poorly with arthroscopic soft tissue procedures alone. In patients with concomitant iliopsoas tendinitis or snap- 


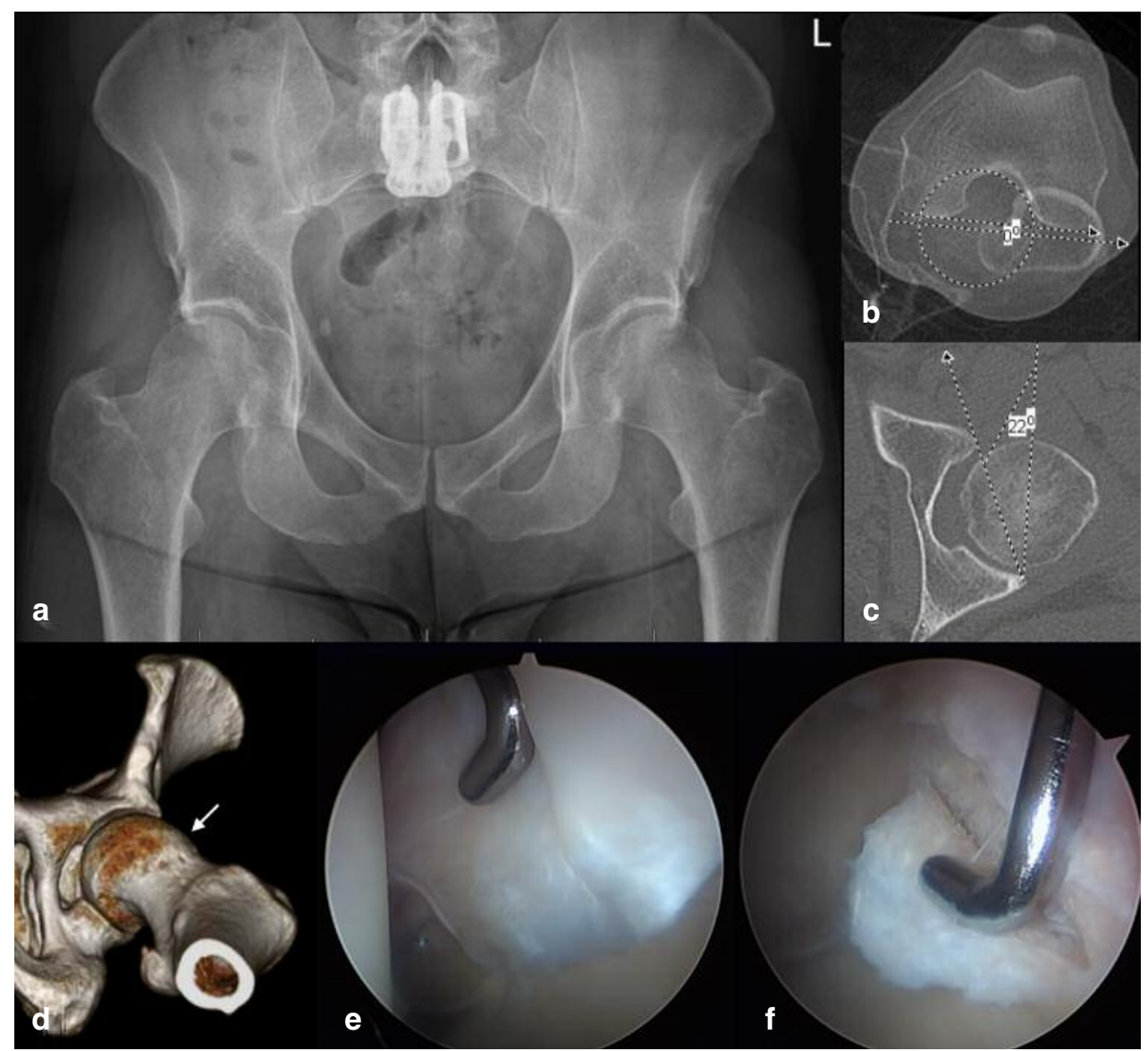

Figure 5 a-f. a) AP radiograph of a pelvis in a 40-year-old male patient presenting with 12-month history of left groin pain and mechanical symptoms, having failed conservative management. His clinical examination is concerning for limited left hip internal rotation of $5^{\circ}$ with the hip at $90^{\circ}$ of flexion, despite have a severely dysplastic acetabulum. Note the positive posterior wall sign with posterior acetabular deficiency which, in conjunction with reduced lateral coverage, represents global acetabular deficiency. b) Axial CT scans of the left hip with overlapping images through the center of the femoral head, the lesser trochanter, and the distal femur. Femoral torsion measures $0^{\circ}$ (normal 10-20 antetorsion), indicating relative retrotorsion, and accounting for the limited internal rotation seen on clinical exam. c) Axial CT scan of the left hip through the center of the femoral head indicating equatorial acetabular version to be $22^{\circ}$ anteversion (normal $15-20^{\circ}$ anteversion). Given the posterior wall deficiency, this value underrepresents the true extent of anterior acetabular deficiency. d) Three-dimensional CT reconstruction of the left hip indicating a large cam lesion in the upper femur (white arrow). e) Arthroscopic image of the left hip demonstrating enlarged and torn labrum consistent with instability. f) Arthroscopic image of the left hip demonstrating full thickness "outside-in" articular flap with a break in the chondrolabral junction consistent with cam-type FAI. The impingement is further exacerbated by the femoral retrotorsion. The pattern of intra-articular injury in this hip supports both a diagnosis of impingement and that of instability.

ping hip syndrome, psoas tenotomy or lengthening procedures should be avoided as these have been shown to contribute to iatrogenic hip instability.

Future studies are necessary to determine long-term outcomes of patients with borderline hip dysplasia treated with a soft tissue-only approach versus osteotomy procedures. In addition, it is necessary to determine which patients benefit from combined arthroscopy and periacetabular osteotomy.

\section{References}

1. Kolb A, Schweiger N, Mailath-Pokorny M, et al. Low incidence of early developmental dysplasia of the hip in universal ultra- sonographic screening of newborns: analysis and evaluation of risk factors. Int Orthop. 2016;40:123-127.

2. Guille JT, Pizzutillo PD, MacEwen GD. Developmental dysplasia of the hip from birth to six months. J Am Acad Orthop Surg. 2000;8:232-242.

3. Padulo J, Oliva F, Frizziero A, Maffulli N. Muscles, Ligaments and Tendons Journal. Basic principles and recommendations in clinical and field science research: 2016 Update. MLTJ. 2016;6(1):1-5.

4. Laplaza FJ, Root L. Femoral anteversion and neck-shaft angles in hip instability in cerebral palsy. J Pediatr Orthop. 1994;14:719-723.

5. Canham CD, Yen YM, Giordano BD. Does femoroacetabular impingement cause hip instability? A systematic review. Arthroscopy. 2016;32:203-208.

6. Larson CM, Stone RM, Grossi EF, Giveans MR, Cornelsen 
GD. Ehlers-Danlos syndrome: Arthroscopic management for extreme soft-tissue hip instability. Arthroscopy. 2015;31:22872294.

7. Ezoe M, Naito M, Inoue T. The prevalence of acetabular retroversion among various disorders of the hip. J Bone Joint Surg Am. 2006;88:372-379.

8. Fujii M, Nakashima Y, Yamamoto T, et al. Acetabular retroversion in developmental dysplasia of the hip. J Bone Joint Surg Am. 2010;92:895-903.

9. Gala L, Clohisy JC, Beaulé PE. Hip dysplasia in the young adult. J Bone Joint Surg Am. 2016;98:63-73.

10. Chadayammuri V, Garabekyan T, Bedi A, et al. Passive hip range of motion predicts femoral torsion and acetabular version. J Bone Joint Surg Am. 2016;98:127-134.

11. Garabekyan T, Ashwell Z, Chadayammuri V, et al. Lateral acetabular coverage predicts the size of the hip labrum. Am J Sports Med. 2016;44:1582-1589.

12. Gupta A, Chandrasekaran S, Redmond JM, et al. Does labral size correlate with degree of acetabular dysplasia? Orthop J Sports Med. 2015;3:2325967115572573.

13. Kim HT, Lee TH, Ahn TY, Jang JH. The fate of DDH hips showing cartilaginous or fibrous tissue-filled joint spaces following primary reduction. J Pediatr Orthop. 2015. [Epub ahead of print].

14. Tréquier C, Baud C, Ferry M, et al. Irreducible developmental dysplasia of the hip due to acetabular roof cartilage hypertrophy. Diagnostic sonography in 15 hips. Orthop Traumatol Surg Res. 2011;97:629-633.

15. Ashwell ZR, Flug J, Chadayammuri V, Pascual-Garrido C, Garabekyan T, Mei-Dan O. Lateral acetabular coverage predicts femoroacetabular cartilage thickness. In Review.

16. Ross JR, Zaltz I, Nepple JJ, Schoenecker PL, Clohisy JC. Arthroscopic disease classification and interventions as an adjunct in the treatment of acetabular dysplasia. Am J Sports Med. 2011;39 Suppl:72S-78S.

17. Martin RL, Palmer I, Martin HD. Ligamentum teres: a functional description and potential clinical relevance. Knee Surg Sports Traumatol Arthrosc. 2012;20:1209-1214.

18. Duplantier NL, McCulloch PC, Nho SJ, Mather RC 3rd, Lewis BD, Harris JD. Hip dislocation or subluxation after hip arthroscopy: A systematic review. Arthroscopy. 2016;32: 1428-1434.

19. Yeung M, Memon M, Simunovic N, Belzile E, Philippon MJ, Ayeni OR. Gross instability after hip arthroscopy: An analysis of case reports evaluating surgical and patient factors. Arthroscopy. 2016;32:1196-1204.e1.

20. Fitzpatrick CK, Steensen RN, Tumuluri A, Trinh T, Bentley J, Rullkoetter PJ. Computational analysis of factors contributing to patellar dislocation. J Orthop Res. 2016;34:444-453.

21. Blakey CM, Field MH, Singh PJ, Tayar R, Field RE. Secondary capsular laxity of the hip. Hip Int. 2010;20:497-504.

22. O'Donnell J, Economopoulos K, Singh P, Bates D, Pritchard $M$. The ligamentum teres test: a novel and effective test in diagnosing tears of the ligamentum teres. Am J Sports Med. 2014;42:138-143.

23. Al Kaissi A, Zwettler E, Ganger R, Schreiner S, Klaushofer K, Grill F. Musculo-skeletal abnormalities in patients with Marfan syndrome. Clin Med Insights Arthritis Musculoskelet Disord. 2013;6:1-9.

24. Beighton P, Horan F. Orthopaedic aspects of the Ehlers-Danlos syndrome. J Bone Joint Surg Br. 1969;51:444-453.

25. Beighton $\mathrm{P}$, Solomon L, Soskolne CL. Articular mobility in an African Population. Ann Rheum Dis. 1973;32:413-418.

26. Naal FD, Hatzung G, Müller A, Impellizzeri F, Leunig M. Validation of a self-reported Beighton score to assess hypermobility in patients with femoroacetabular impingement. Int Orthop. 2014;38:2245-2250.

27. Wiberg G. Studies on dysplastic acetabula and congenital subluxation of the hip joint. With special reference to the complication of osteoarthritis. Acta Chir Scand.1939;83(Suppl 58):28-38.

28. Ogata S, Moriya H, Tsuchiya K, Akita T, Kamegaya M, Someya M. Acetabular cover in congenital dislocation of the hip. J Bone Joint Surg Br. 1990;72:190-196.

29. Chadayammuri V, Garabekyan T, Jesse MK, et al. Measurement of lateral acetabular coverage: a comparison between CT and plain radiography. J Hip Preserv Surg. 2015;2:392400.

30. Clohisy JC, Carlisle JC, Beaulé PE, et al. A systematic approach to the plain radiographic evaluation of the young adult hip. J Bone Joint Surg Am. 2008;90 Suppl 4:47-66.

31. Frank RM, Harris JD, Erickson BJ, et al. Prevalence of femoroacetabular impingement imaging findings in asymptomatic volunteers: A systematic review. Arthroscopy. 2015;31: 1199-1204.

32. Larson CM, Moreau-Gaudry A, Kelly BT, et al. Are normal hips being labeled as pathologic? A CT-based method for defining normal acetabular coverage. Clin Orthop Relat Res. 2015;473:1247-1254.

33. Lequesne M, de Seze. [False profile of the pelvis. A new radiographic incidence for the study of the hip. Its use in dysplasias and different coxopathies]. Rev Rhum Mal Osteoartic. 1961;28:643-652.

34. Needell SD, Borzykowski RM, Carreira DS, Kozy J. CT falseprofile view of the hip: a reproducible method of measuring anterior acetabular coverage using volume CT data. Skeletal Radiol. 2014;43:1605-1611.

35. Jia J, Li J, Zhang L, Zhao Q, Liu X. Three dimensional-CT evaluation of femoral neck anteversion, acetabular anteversion and combined anteversion in unilateral DDH in an early walking age group. Int Orthop. 2012;36:119-124.

36. Fujii M, Nakashima Y, Sato T, Akiyama M, Iwamoto Y. Pelvic deformity influences acetabular version and coverage in hip dysplasia. Clin Orthop Relat Res. 2011;469:1735-1742.

37. Tannast M, Zheng G, Anderegg C, et al. Tilt and rotation correction of acetabular version on pelvic radiographs. Clin Orthop Relat Res. 2005;438:182-190.

38. Yin $Y$, Zhang $L$, Hou Z, et al. Measuring femoral neck torsion angle using femoral neck oblique axial computed tomography reconstruction. Int Orthop. 2016;40:371-376.

39. Tomczak RJ, Guenther KP, Rieber A, Mergo P, Ros PR, Brambs HJ. MR imaging measurement of the femoral antetorsional angle as a new technique: comparison with CT in children and adults. AJR Am J Roentgenol. 1997;168:791-794.

40. Dunn DM. Anteversion of the neck of the femur; a method of measurement. J Bone Joint Surg Br. 1952;34-B:181-186.

41. Datir A, Xing M, Kang J, et al. Diagnostic utility of MRI and MR arthrography for detection of ligamentum teres tears: a retrospective analysis of 187 patients with hip pain. AJR Am J Roentgenol. 2014;203:418-423.

42. Hunt D, Prather H, Harris Hayes M, Clohisy JC. Clinical outcomes analysis of conservative and surgical treatment of patients with clinical indications of prearthritic, intra-articular hip disorders. PM R 2012;4:479-487.

43. Emara K, Samir W, Motasem el H, Ghafar KA. Conservative treatment for mild femoroacetabular impingement. J Orthop Surg (Hong Kong). 2011;19:41-45.

44. Wall PD, Fernandez M, Griffin DR, Foster NE. Nonoperative treatment for femoroacetabular impingement: a systematic review of the literature. PM R 2013;5:418-426.

45. Abate M, Scuccimarra T, Vanni D, Pantalone A, Salini V. Femoroacetabular impingement: is hyaluronic acid effective? Knee Surg Sports Traumatol Arthrosc. 2014;22:889-892.

46. Khan W, Khan M, Alradwan H, Williams R, Simunovic N, Ayeni OR. Utility of intra-articular hip injections for femoroacetabular impingement: A systematic review. Orthop J Sports Med. 2015;3:2325967115601030. 
47. Levy DM, Grzybowski J, Salata MJ, Mather RC 3rd, Aoki SK, Nho SJ. Capsular plication for treatment of iatrogenic hip instability. Arthrosc Tech. 2015;4:e625-e630.

48. Philippon MJ. New frontiers in hip arthroscopy: the role of arthroscopic hip labral repair and capsulorrhaphy in the treatment of hip disorders. Instr Course Lect. 2006;55:309-316.

49. Mei-Dan O, Garabekyan T, McConkey M, Pascual-Garrido C. Arthroscopic anterior capsular reconstruction of the hip for recurrent instability. Arthrosc Tech. 2015;4:e711-e715.

50. Fabricant PD, Bedi A, De La Torre K, Kelly BT. Clinical outcomes after arthroscopic psoas lengthening: the effect of femoral version. Arthroscopy. 2012;28:965-971.

51. Harris JD, Gerrie BJ, Lintner DM, Varner KE, McCulloch PC. Microinstability of the hip and the splits radiograph. Orthopedics. 2016;39:e169-e175.

52. Sansone M, Ahldén $M$, Jónasson $P$, Swärd L, Eriksson $T$, Karlsson J. Total dislocation of the hip joint after arthroscopy and ileopsoas tenotomy. Knee Surg Sports Traumatol Arthrosc. 2013;21:420-423.

53. Nepple JJ, Philippon MJ, Campbell KJ, et al. The hip fluid sealPart II: The effect of an acetabular labral tear, repair, resection, and reconstruction on hip stability to distraction. Knee Surg Sports Traumatol Arthrosc. 2014;22:730-736.

54. Domb BG, Stake CE, Lindner D, El-Bitar Y, Jackson TJ. Arthroscopic capsular plication and labral preservation in borderline hip dysplasia: two-year clinical outcomes of a surgical approach to a challenging problem. Am J Sports Med. 2013; 41:2591-2598.

55. Uchida S, Utsunomiya $\mathrm{H}$, Mori $\mathrm{T}$, et al. Clinical and radiographic predictors for worsened clinical outcomes after hip arthroscopic labral preservation and capsular closure in developmental dysplasia of the hip. Am J Sports Med. 2016;44:2838.

56. Kosuge D, Yamada N, Azegami S, Achan P, Ramachandran $M$. Management of developmental dysplasia of the hip in young adults: current concepts. Bone Joint J. 2013;95-B:732737.

57. Steppacher SD, Tannast M, Ganz R, Siebenrock KA. Mean 20-year follow-up of Bernese periacetabular osteotomy. Clin Orthop Relat Res. 2008:466:1633-1644.

58. Kumar D, Bache CE, O'Hara JN. Interlocking triple pelvic osteotomy in severe Legg-Calvé-Perthes disease. J Pediatr Orthop. 2002;22:464-470.

59. Lodhia P, Chandrasekaran S, Gui C, Darwish N, Suarez-Ahedo C, Domb BG. Open and arthroscopic treatment of adult hip dysplasia: A systematic review. Arthroscopy. 2016;32:374-383.
60. Troelsen A, Elmengaard B, Søballe K. Medium-term outcome of periacetabular osteotomy and predictors of conversion to total hip replacement. J Bone Joint Surg Am. 2009;91:21692179.

61. Osawa Y, Hasegawa Y, Seki T, Amano T, Higuchi Y, Ishiguro N. Significantly poor outcomes of total hip arthroplasty after failed periacetabular osteotomy. J Arthroplasty. 2016;31: 1904-1909.

62. Kalore NV, Cheppalli SP, Daner WE 3rd, Jiranek WA. Acetabular dysplasia in middle-aged patients: Periacetabular osteotomy or total hip arthroplasty? J Arthroplasty. 2016;31: 1894-1898.

63. Gordon JE, Pappademos PC, Schoenecker PL, Dobbs MB Luhmann JS. Diaphyseal derotational osteotomy with intramedullary fixation for correction of excessive femoral anteversion in children. J Pediatr Orthop. 2005;25:548-553.

64. Payne LZ, DeLuca PA. Intertrochanteric versus supracondylar osteotomy for severe femoral anteversion. J Pediatr Orthop. 1994;14:39-44

65. Schmidt D, Arnold AS, Carroll NC, Delp SL. Length changes of the hamstrings and adductors resulting from derotational osteotomies of the femur. J Orthop Res. 1999;17:279-285.

66. Kaya M, Suzuki T, Emori M, Yamashita T. Hip morphology influences the pattern of articular cartilage damage. Knee Surg Sports Traumatol Arthrosc. 2016;24:2016-2023.

67. Tamura S, Nishii T, Takao M, Sakai T, Yoshikawa H, Sugano N. Differences in the locations and modes of labral tearing between dysplastic hips and those with femoroacetabular impingement. Bone Joint J. 2013;95-B:1320-1325.

68. Ganz R, Leunig M, Leunig-Ganz K, Harris WH. The etiology of osteoarthritis of the hip: an integrated mechanical concept. Clin Orthop Relat Res. 2008;466:264-272.

69. Nawabi DH, Degen RM, Fields KG, et al. Outcomes after arthroscopic treatment of femoroacetabular impingement for patients with borderline hip dysplasia. Am J Sports Med. 2016;44:1017-1023.

70. Botser IB, Martin DE, Stout CE, Domb BG. Tears of the ligamentum teres: prevalence in hip arthroscopy using 2 classification systems. Am J Sports Med. 2011;39 Suppl:117S-125S.

71. Cerezal L, Carro LP, Llorca J, et al. Usefulness of MR arthrography of the hip with leg traction in the evaluation of ligamentum teres injuries. Skeletal Radiol. 2015;44:1585-1595.

72. Garabekyan T, Chadayammuri V, Pascual-Garrido C, MeiDan O. All-arthroscopic ligamentum teres reconstruction with graft fixation at the femoral head-neck junction. Arthrosc Tech. 2016;5:e143-e147. 